\title{
Precision HIV care: responding to old questions and meeting new challenges
}

\author{
Todd Hulgan ${ }^{1,3}$, Chandravanu Dash²,3, David W Haas ${ }^{1,3,4}$ \& Elizabeth J Phillips*,1,3,5 \\ ${ }^{1}$ Department of Medicine, Division of Infectious Diseases, Vanderbilt University Medical Center, Nashville, TN 37232, USA \\ ${ }^{2}$ Department of Biochemistry \& Cancer Biology, Center for AIDS Health Disparities Research, Meharry Medical College, Nashville, \\ TN 37208, USA \\ ${ }^{3}$ Tennessee Center for AIDS Research, Nashville, TN 37232, USA \\ ${ }^{4}$ Department of Internal Medicine, Meharry Medical College, Nashville, TN 37208, USA \\ ${ }^{5}$ John A. Oates Institute for Experimental Therapeutics, Vanderbilt University School of Medicine, Nashville, TN 37232, USA \\ *Author for correspondence: elizabeth.j.phillips@vanderbilt.edu
}
"Personalized HIV care approaches that evolved based on the unmet needs in the past can serve as roadmaps for future precision HIV care that extends beyond pharmacogenetics."

First draft submitted: 1 August 2018; Accepted for publication: 26 August 2018; Published online: 16 October 2018

Keywords: HIV • pharmacogenetics • precision medicine

\begin{abstract}
Advances in the care of persons living with HIV (PLWH) and improvements in clinical outcomes over the last two decades have been substantial. In the early days of antiretroviral treatment (ART), treatment success was challenging due to high pill burdens, toxicities, drug interactions and viral resistance. These factors drove the development of simplified and well-tolerated single-tablet regimens, and clinical studies that supported earlier treatment. Basic HIV-related research has also driven seminal discoveries in retroviral biology and human immunology, and has led at least one proof-of-concept example [1] to explicit discussions [2] and early-stage clinical trials [3] of strategies to cure HIV infection. Despite scientific progress and justifiable optimism, many PLWH in diverse global settings do not fully realize the benefits of ART, and many who achieve virologic suppression with ART still suffer from prior and current ART toxicity, as well as persistent immune dysregulation [4,5]. The opportunity for precision medicine to improve HIV care is still highlighted by the inconsistent access to and success of treatment, and in manifestations of these sequelae of infection, treatment and non-HIV co-morbidities across populations. In addition, personalized approaches are relevant to new frontiers of HIV science that include developing an effective preventative vaccine as well as a curative intervention.
\end{abstract}

\section{What is precision HIV care?}

Precision medicine has been variously defined. Many have considered it as primarily dealing with human genetic variation. More recent definitions provide an expanded and multidisciplinary framework for understanding precision medicine. The NIH Precision Medicine Initiative working group defined precision medicine as "... an approach to disease treatment and prevention that seeks to maximize effectiveness by taking into account individual variability in genes, environment, and lifestyle [6]." This definition underscores challenges with optimizing HIV care that require consideration of both viral and host biology, and social contexts. Key pathogenic discoveries that ultimately translated to routine clinic care include the use of $H L A-B^{*}$ 57:01 as a genetic screening marker prior to abacavir use to prevent a potentially life-threatening hypersensitivity syndrome [7]. This provided a new roadmap for the use of a genetic screening marker to predict and prevent a specific toxicity. Since 2008, HLA-B*57:01 testing is routinely performed prior to abacavir exposure in most settings as part of guideline-based practice [7]. CCR5 biology is related to HIV transmission and was a target for development of a new class of ART [8]. HIV has long been at the leading edge of genetics-based precision medicine, as exemplified by decades of 'individualized' ART based on sophisticated viral genotyping, phenotyping and tropism assays. Personalized HIV care approaches that evolved based on the unmet needs in the past can serve as roadmaps for future precision HIV care that extends beyond pharmacogenetics.
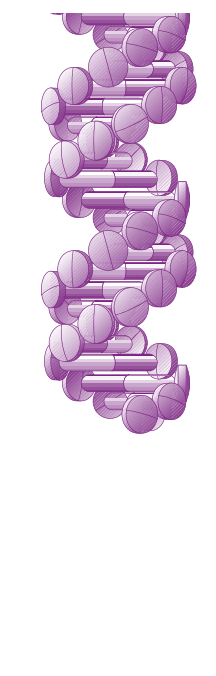


\section{Categories \& priorities of precision HIV care}

To expand precision HIV care beyond pharmacogenetics, it is helpful to consider domains not typically viewed as part of 'personalized medicine'. This is especially true for basic science research given the recent discovery and development of cutting-edge technologies that have shed new light on virus and host biology, such as CRISPR/Cas9 [9]. Fundamental research into HIV is by nature 'precise' in its focus on viral or immunologic molecular mechanisms. Using viral gene variation to guide decision-making and precision care where resources allow is well established in HIV care [10]. Translational and clinical approaches to precision HIV care will be enhanced by collaborative, transdisciplinary, hypothesis-testing research. For example, studies to understand HIVmediated immune activation and inflammation can lead to new interventions that target interindividual differences in these responses and/or end-organ phenotypes. A multidisciplinary program in precision HIV care might include a population-to-bench-to-bedside approach that incorporates epidemiologic observations and expected implementation needs, together with laboratory experiments focused on design of targeted interventions. Examples have included observations regarding the aforementioned CCR5 that led to new understanding of pathogenesis as well as development of a therapeutic CCR5 antagonist. Studies in basic immunology fostered new hypotheses, translational discoveries and ultimately clinical trials and cost-effectiveness analysis of $H L A-B^{*} 57: 01$ screening to prevent abacavir hypersensitivity reactions. These are 'gold-standard' precision HIV care approaches. Challenges remain in areas where genetic risk factors are not 'monogenic' (as with CCR5 homozygous allele variation), or where the predictive value of genetic testing is far less than $100 \%$. These prototypical examples also contrast with a vision of precision HIV care that moves beyond host genetics.

\section{Responding to old questions about precision HIV care}

As a framework to consider successes, gaps and future directions for precision HIV care, we use the framework of questions regarding personalized medicine posed by Joyner and Paneth in 2015 [11]. In the following sections, we attempt to answer select questions from that Viewpoint article, and reframe them in the context of personalized HIV care. Responses to these questions are also relevant for considering precision healthcare beyond HIV.

\section{Can precision HIV care predict disease risk?}

The answer is 'yes'. For example, a subgroup of individuals who are highly resistant to acquiring HIV was identified more than 20 years ago [12]. These persons remained uninfected despite repeated HIV exposures due to homozygosity for a shared CCR5 32 base-pair deletion [8]. Rates of progression to AIDS with untreated HIV infection have been associated with other immunogenetic variants, including specific $H L A$ and KIR types as well as heterozygosity for CCR5 $32[13]$.

\section{Will precision HIV care include gene-based drug targeting \& development?}

This answer is also 'yes'. Following discovery of the CCR $5 \Delta 32$ polymorphism described above, drugs were developed to block HIV access to the CCR5 molecule on host cells. This ultimately resulted in the US FDA-approved CCR5 entry inhibitor, maraviroc. Of note, the only known cure of established HIV infection to date was in Timothy Brown ('the Berlin patient'), following treatment of his leukemia with bone marrow transplantation from a specifically chosen CCR5 32 donor in 2007 [1]. The ability of abacavir to be widely prescribed for HIV was possible only by coupling this drug with genotyping to exclude the presence of $H L A-B^{*} 57: 01$ [7].

\section{How will electronic medical records contribute to precision HIV care?}

This question has been partially answered. Electronic medical records have contributed substantially inasmuch as observational HIV outcomes research in resource-rich countries has been driven by collaborative analyses of retrospective cohort-based data. In HIV research, there is a long tradition of single-site, multisite and even multicountry cohort consortia to address outcomes of HIV infection, its therapy and complications, which has often depended on data extracted from electronic medical records. These studies have shaped our understanding of predictors of outcomes in populations with HIV and provided a foundation for precision HIV care.

What kinds of studies should be performed in the future to address gaps in precision HIV care? This question has as many answers as there are stakeholders. The HIV basic scientist may see fundamental mechanistic studies as critical to understand the biology that underlies interindividual variability. The HIV clinician may argue that definitive clinical efficacy trials of precision HIV care strategies are the sine qua non. The HIV 
implementation scientist may envision pragmatic trials as the gold standard to affirm feasibility of precision HIV care interventions. The public health practitioner may favor studies demonstrating population-level benefits of precision HIV care approaches. Policy makers may ask for cost-effectiveness analyses to assess the impact of precision HIV care on the overall healthcare system or society. All of these responses are valid and will lead to a more comprehensive understanding of precision HIV care, including its feasibility and challenges. PLWH and other community-based stakeholders might answer the question differently. In a community engagement studio for the Tennessee Center for AIDS Research, organized by the Meharry-Vanderbilt Community Engaged Research Core, community members primarily asked questions about how to raise awareness and communicate expectations about precision HIV care, and how to ensure privacy and confidentiality in precision HIV care research. We conclude that to best address gaps in precision HIV care, future studies should have multidisciplinary leadership that includes community representatives, and address multiple needs at once. As noted above, studies of HLA-B*57:01 testing for abacavir hypersensitivity provide a translational roadmap. These studies routinely incorporated biologic testing that contributed to mechanistic discoveries, collected information used in cost-effectiveness analyses and emphasized pragmatic approaches that could be implemented in diverse settings and populations [7].

\section{How will precision HIV care affect costs of care?}

The importance of cost-effectiveness of precision HIV care was noted above. HLA-B*57:01 testing before prescribing abacavir was shown to be cost-effective in populations with different baseline prevalence rates [7]. Examples of precision population health in HIV include recommending pretreatment HIV resistance testing only when community prevalence of transmitted resistance reaches levels that meet cost-effective levels of quality-adjusted life-years gained [14], and recent evidence suggesting that pretreatment testing for HIV integrase resistance may never reach cost-effective thresholds [15].

\section{Where is the public health benefit of precision HIV care?}

In resource-rich settings, HIV care has been transformed by the introduction of tolerable regimens that are prescribed as early as possible after HIV diagnosis. Beyond individual health outcomes, benefits have included decreased HIV transmission [16]. Targeting scarce public health resources to those at greatest risk will always be necessary. Public health benefits could come from the use of biological (e.g., genetics and biomarkers) or sociobehavioral (e.g., geographic location and social networks) precision HIV care strategies to stratify individuals by their likelihood of benefitting from pre-exposure prophylaxis (PrEP). Cost-effectiveness of PrEP depends heavily on the baseline transmission risk of the population and subsequent medication adherence [17]. Precision HIV care strategies that incorporate these factors to enhance PrEP implementation could have considerable public health impact.

\section{Future challenges for precision HIV care}

What are the next questions in precision HIV care? Long-acting ART represents an attractive option for individuals and populations with adherence challenges. Potential risk of toxicity and resistance with prolonged exposure to long-acting drug formulations make a precision approach even more important. Identifying patients at the greatest risk for treatment failure, either due to biologic (e.g., pharmacogenetic variants) or sociobehavorial (e.g., loss to follow-up) factors, may inform the implementation of these drugs. Future interventions that may include effective HIV vaccines and promising cure strategies will also be targets for precision care research.

\section{Conclusion}

From its onset, HIV has provided both the imperative for and examples of precision care models that incorporate genetic and ecological information of the virus and host. Translational success stories, such as the discovery of new drug targets and the routine use of pretreatment $H L A-B^{*} 57: 01$ screening, are key examples of precision care that will continue to be used as models within and outside the field of HIV for years to come. Over the last 35 years, there has been an evolution from individual precision care to population-based treatment and preventative approaches that have reduced the global burden of HIV. Future strategies will continue to embody precision care approaches aimed at optimizing the care of the PLWH, while at a population level aiming to eradicate new transmission and HIV as a disease. 


\section{Acknowledgements}

This work was informed by our experiences leading a scientific working group of the Tennessee Center for AIDS Research at our institutions and the Tennessee Department of Health. The authors thank the Tennessee Center for AIDS Research Directors (Simon Mallal, MBBS, Duane Smoot, MD. and James Hildreth, MD, PhD), Executive Committee members and Program Managers (Vickie Myers and Heather Burgess) for their support and feedback, and the organizers and participants involved in the Personalized HIV Care Community Engagement Studio.

\section{Financial \& competing interests disclosure}

This work was supported by the NIH-funded Tennessee Center for AIDS Research (P30 Al110527) and in part by CTSA award no. UL1 TR002243 from the National Center for Advancing Translational Sciences. Additional support for this work was provided by NIH grants Al077505 (to D Haas), Al22960 and MD00756 (to C Dash), and GM115305, Al139021 and Al136815 (to E Phillips). Its contents are solely the responsibility of the authors and do not necessarily represent official views of the National Center for Advancing Translational Sciences or the NIH. The authors have no other relevant affiliations or financial involvement with any organization or entity with a financial interest in or financial conflict with the subject matter or materials discussed in the manuscript apart from those disclosed.

No writing assistance was utilized in the production of this manuscript.

\section{References}

1. Hutter G, Nowak D, Mossner M et al. Long-term control of HIV by CCR5 Delta32/Delta32 stem-cell transplantation. N. Engl. J. Med. 360(7), 692-698 (2009).

2. Liu C, Ma X, Liu B, Chen C, Zhang H. HIV-1 functional cure: will the dream come true? BMC Med. 13, 284 (2015).

3. Tebas P, Stein D, Tang WW et al. Gene editing of CCR5 in autologous CD4 T cells of persons infected with HIV. N. Engl. J. Med. 370(10), 901-910 (2014).

4. Hunt PW, Lee SA, Siedner MJ. Immunologic biomarkers, morbidity, and mortality in treated HIV infection. J. Infect. Dis. 214(Suppl. 2), S44-S50 (2016).

5. Srinivasa S, Grinspoon SK. Metabolic and body composition effects of newer antiretrovirals in HIV-infected patients. Eur. J. Endocrinol. 170(5), R185-R202 (2014).

6. The Precision Medicine Initiative Cohort Program - building a research foundation for 21st century medicine. Group PMIPW 17 September 2015. https:/www.nih.gov/sites/default/files/research-training/initiatives/pmi/pmi-working-group-report-20150917-2.pdf

7. Phillips E, Mallal S. Successful translation of pharmacogenetics into the clinic: the abacavir example. Mol. Diagn. Ther. 13(1), 1-9 (2009).

8. Brelot A, Chakrabarti LA. CCR5 revisited: how mechanisms of HIV entry govern AIDS pathogenesis. J. Mol. Biol. 430(17), 2557-2589 (2018).

9. Saayman S, Ali SA, Morris KV, Weinberg MS. The therapeutic application of CRISPR/Cas9 technologies for HIV. Expert Opin. Biol. Ther. 15(6), 819-830 (2015).

10. Clutter DS, Jordan MR, Bertagnolio S, Shafer RW. HIV-1 drug resistance and resistance testing. Infect. Genet. Evol. 46, 292-307 (2016).

11. Joyner MJ, Paneth N. Seven questions for personalized medicine. JAMA 314(10), 999-1000 (2015).

12. Samson M, Libert F, Doranz BJ et al. Resistance to HIV-1 infection in caucasian individuals bearing mutant alleles of the CCR-5 chemokine receptor gene. Nature 382(6593), 722-725 (1996).

13. Fellay J, Shianna KV, Ge D et al. A whole-genome association study of major determinants for host control of HIV-1. Science 317(5840), 944-947 (2007).

14. Sax PE, Islam R, Walensky RP et al. Should resistance testing be performed for treatment-naive HIV-infected patients? A cost-effectiveness analysis. Clin. Infect. Dis. 41(9), 1316-1323 (2005).

15. Koullias Y, Sax PE, Fields NF, Walensky RP, Hyle EP. Should we be testing for baseline integrase resistance in patients newly diagnosed with human immunodeficiency virus? Clin. Infect. Dis. 65(8), 1274-1281 (2017).

16. Rodger AJ, Cambiano V, Bruun T et al. Sexual activity without condoms and risk of HIV transmission in sero different couples when the HIV-positive partner is using suppressive antiretroviral therapy. JAMA 316(2), 171-181 (2016).

17. Schackman BR, Eggman AA. Cost-effectiveness of pre-exposure prophylaxis for HIV: a review. Curr. Opin. HIV AIDS 7(6), 587-592 (2012). 